\title{
RESPONDING TO COVID-19: RAPID ORGANISATIONAL CHANGE IN ONLINE CPD PRODUCTION A CASE REPORT
}

\author{
Dirk Pilat $^{1,2}$ and David Cameron ${ }^{3,4}$ \\ Royal College of General Practitioners, London, UK \\ ${ }^{I} \mathrm{Dr}, \mathrm{FRCGP}$, PgDipGp (Otago), BSc (Open), MA ODE (Open) \\ ${ }^{2}$ Medical Director for eLeaning and Accreditation \\ ${ }^{3} \mathrm{Dr}, \mathrm{MA}$ (Oxon.), PhD \\ ${ }^{4}$ Assistant Director, Professional Development
}

\begin{abstract}
Over only a few weeks in March 2020 the COVID-19 pandemic changed health care delivery in the United Kingdom completely - not least in general practice, where over 1 million patients are already treated every day. To reduce viral spread, practices introduced remote doctor-led triage and face-to-face consultations where converted almost exclusively to telephone or video. Guidance on the management of this new, emerging disease changed frequently, so the UK's Royal College of General Practitioners (RCGP) took the strategic decision to direct a large part of the College's staff and resources to provide general practitioners (GPs) with online continuous professional development (CPD) resources around COVID-19 and the new ways of working. In this case report we describe how the RCGP's online learning team responded to the critical imperatives of the pandemic both within project management and educational practice and created a COVID-19 resource hub, covering all aspects of general practice during the pandemic.
\end{abstract}

\section{KEYWORDS}

Continuous Professional Development, e-Learning, COVID-19, Organisational Change, General Practice

\section{INTRODUCTION}

In December 2019 the city of Wuhan, capital of Hubei province in China, became the centre of an outbreak of pneumonia of unknown origin, with most of the initial patients working or living around the local Huanan seafood wholesale market. In the early stages of this pneumonia, severe acute respiratory infection symptoms occurred, with some patients rapidly developing acute respiratory distress syndrome (ARDS), acute respiratory failure and subsequent death. Named "Novel Coronavirus Disease 2019" (COVID-19) by the World Health Organization (WHO), the disease was identified to be caused by the severe acute respiratory syndrome coronavirus 2 (SARS-CoV-2) (Fei Zhou, 2020). Even though the Chinese central government introduced a quarantine of previously unheard severity and scaled up inpatient heath care in Hubei province, SARS-CoV-2 nevertheless spread rapidly across the world, with the first confirmed cases in the UK identified late January 2020 (Moss, Barlow, Easom, Lillie, \& Samson, 2020).

During the first week of March the outbreak had spread across the United Kingdom, with 200 confirmed cases and one fatality. The Royal College of General Practitioners (RCGP), the professional body for family doctors in the UK, recognised there was an immediate need for support and guidance for its members who were facing an unprecedented challenge. A strategic decision was taken to focus a large part of the College's staff and resources onto providing this support and guidance, with immediate effect. This included summaries of guidance released by national governments across the UK and related agencies; policy positions developed to support the profession, for example on the issues of Personal Protective Equipment (PPE) and testing; a newly-created discussion forum to allow members of the College to ask and respond to queries and initiate dialogue with the College leadership; and an online repository, or Hub, of resources and learning materials to support GPs' (and other primary care professionals') professional practice in managing all aspects of the COVID-19 pandemic. The development of the 'Resource Hub' is the subject of this paper. 
A small team of professional staff and clinicians was drawn together with a remit to create the first iteration of the Resource Hub within a few days. This required the development of new processes for resource development and quality assurance, the secondment of clinical expertise from across and beyond the College, as well as staff working in different ways (e.g. event managers shifting to webinar development).

The fast-changing nature of the knowledge base for COVID-19 required a significantly accelerated review and update process for any new resources that were published - in some cases, updates were required on a daily basis as rapid reviews were published by research institutions. This case report aims to outline how the RCGP adapted to and addressed the challenge of providing essential COVID-19 continuing professional development (CPD) resources for the United Kingdom's primary care sector during a critical period of professional challenge and uncertainty.

\section{THE ROLE OF ONLINE CPD IN UK GENERAL PRACTICE}

Patients and the wider public rightly expect the highest degree of professionalism from their physicians. The concept of professionalism encompasses a range of characteristics, including ethics, values, attitudes, judgement, knowledge, skills and 'meta-skills' (Morrow, 2014). The notion of the professional, and professional practice, relies on the ongoing maintenance of domain-specific knowledge and skills; continuing professional development (CPD) is therefore an intrinsic aspect of professionalism, best considered as a 'state of mind' or 'intellectual alignment' rather than a series of discrete events or resources (Cameron \& Grant, 2017). Thus, society and the profession itself demand that family doctors not only fulfil the traditional roles of healer and source of comfort, but also operate on the basis of the latest scientific expertise and maintain the highest standards in education, training and practice. The complexity of modern medicine makes this demand ever more difficult to fulfil for the practising doctor, who has to balance his workday between patient contact, administrative tasks and CPD. Whilst newly qualified doctors are certified as safe to practice, the sheer volume of domain knowledge in medicine, and the pace of its development and revision (Densen, 2011), ensure that CPD is a career-long endeavour which is vital for patient safety. This requires both responding to educational needs that arise during patient contact, and being continuously up to date with the ever-shifting evidence base for effective diagnostic and therapeutic methods (General Medical Council (UK), 2012). Family doctors, or general practitioners (GPs), face a particular challenge in their specialism which requires a very broad range of knowledge across many areas of medicine, as exemplified by the GP Curriculum (RCGP, 2020).

A recent survey showed that GPs are using online resources for CPD often and that these are well accepted, valued and likely driven by the need to meet users' identified learning needs and the positive influence of GP appraisal and revalidation (Cunningham, Alexander, Luty, \& Zlotos, 2019). However, attitudinal differences to online CPD resource utilisation between different GP groups and technical barriers are still present: frequent internet users and doctors under 45 were shown to more comfortable using online resources and finding the experience more enjoyable and cost-effective, compared to low volume users (MacWalter, McKay, \& Bowie, 2016).

The rapid emergence of a global pandemic caused by a novel coronavirus brought into sharp focus the need for GPs to develop and maintain their professional knowledge and skills. Working on the frontline of the national (and global) collective effort against COVID-19, GPs needed to be able to access, assimilate and put into practice a wide range of new information and guidance, which was emerging and changing on a daily basis. This included clinical knowledge related to the presentation, epidemiology and treatment of the disease, but also the necessary changes to traditional ways of working that were required, including patient consultation, practice management and end of life care.

Fortunately, online and e-learning CPD resources can contribute to the rapid upskilling of a professional workforce. E-learning offers a wide variety of formats to encompass a range of learning modes and provides the opportunity for dual coding; this variety also improves the accessibility of learning episodes which can be consumed at convenient times and settings (e.g. short audio podcasts) (VanNieuvenborg, Goossens, De Lepeleire, \& Schoenmakers, 2016). E-learning is therefore particularly suitable to meet the professional learning needs of GPs in the context of an emerging healthcare crisis; as learners, they are self-directed and highly motivated to learn, whilst also extremely time-poor and in need of immediate, relevant and results-oriented knowledge and skills. 


\section{THE RCGP'S ONLINE CPD PROVISION}

The Royal College of General Practitioners was founded in 1952 as an academic body to support good standards of practice, education and research. It represents and supports general practitioners (GPs) on licensing, education, training, research and clinical standards. It sets the curriculum and training needs for GP trainees, holds the mandatory qualification exams and runs a CPD scheme for its 57,000 members (Royal College of General Practitioners, 2012). As part of its commitment to CPD it has been running e-learning programmes since 2008, with various methods of content development, quality assurance and delivery.

\subsection{Essential Knowledge Updates (EKU)}

The Essential Knowledge Updates (EKU) programme was initiated in 2008 to provide GPs with a quick and accessible way to update their knowledge on new evidence relevant to primary care, to encourage effective application of that knowledge in clinical practice, and to enhance their skills and ultimately patients' experience and care. EKU e-learning modules are released on a quarterly basis and are authored by practising GPs under the supervision of an EKU Fellow with experience in effective online learning development. The topics for each individual update are chosen from a literature search on primary care journals and resources by one of the RCGP's information scientists and is reduced by the lead GP for the programme from ca 300 papers and guidelines covering the last six months to about fifty, only choosing papers that are applicable for front-line general practice. These are then voted upon by a panel of senior academic general practitioners. Each new Update undergoes a five-step quality assurance process, including literature reviews and topic refinement on the basis of frontline relevancy and content revision by a group of senior academic GPs. EKU modules are accessed by about 38,000 GPs per year. (Pilat, Content development for 72,000 Learners: an online learning environment for general practitioners; a case study, 2016).

\subsection{Online Learning Environment}

The Online Learning Environment (OLE) is a collection of distance and blended CPD courses. Delivered via the whole palette of e-learning including screencasts, podcasts, blogs and modules, it produces content written by GPs for GPs, mapped against the GP curriculum. Responding to the needs of the GP workforce, it produces e-learning based on areas identified by both the learners and current academic literature in which primary care is struggling, such as early cancer diagnosis. The use of a combination of different media elements that cater to the various learning preferences reinforces the take home messages, promotes reflection, identifies knowledge gaps and helps to demonstrate learning. Working in close cooperation with its partners in academia, public health, non-governmental and commercial organisations it has found acclaim both from its learners and external organisations such as the National Institute for Health and Care Excellence (NICE) and has won two national eLearning awards.

With currently over 120,000 registered learners, its content is consistently rated highly by its users. (Royal College of General Practitioners eLearning Department, 2017). The success of the OLE is closely associated with its content development model which guarantees content accuracy and appropriateness for its audience: each course follows a six step quality assurance workflow, starting with a scoping call or meeting of a peer review group, in which interested general practitioners, hospital specialists, a GP author and a GP editor decide on the content and produce a scoping document. After the scoping document is signed off by the peer review group, a GP author in cooperation with an eLearning fellow produces a first draft which is forwarded to the group for the first round of comments. After incorporation of comments and suggestions, the course production moves on to reflect the instructional design, after which it undergoes a second round of peer reviews. The course is then adapted to incorporate additional comments from the peer review group, thereafter the course is built before it is quality assured by the medical director for eLearning and launched (Royal College of General Practitioners eLearning Department, 2017). While tried and tested over ten years, this model unfortunately was not responsive enough for the challenge that COVID-19 presents, as the involvement of external stakeholders often leads to prolonged periods of discussion around the content during peer review, resulting in typical turnaround time of four months from inception to release. 


\section{ONLINE CPD IN RESPONSE TO THE COVID-19 PANDEMIC}

The COVID-19 pandemic emerged at a moment when general practice in the UK is facing a number of structural and demographic challenges, including increased demand due to an ageing patient load with an ever increasing range of comorbidities (Pilat, Access and the great socioeconomic divide, 2015). As an urgent and spreading threat whose clinical and epidemiological characteristics are still being documented, upskilling the primary care workforce to address the challenge of COVID-19 was urgently needed. In addition, public health measures imposed by the UK Government, including social distancing, isolation and shielding, required an immediate shift in ways of working, with in-person consultation almost entirely replaced by remote (phone or video) consultation in a very short timeframe. Clinicians were thus faced with a new disease and a new way of interacting with patients (Greenhalgh, Covid-19: a remote assessment in primary care, 2020). It was therefore necessary to create online resources to not only to keep members of the primary care team informed about the rapidly changing evidence base but also introduce them to best practice in new ways of working. An example for how the evidence base evolved was the use of the Roth Score in suspected COVID-19 presentations: initially, it was thought to be a helpful tool to assess patients with breathlessness remotely during a phone consultation. Guidance on its use was rapidly reproduced and circulated on social media, professional distribution lists and through both local and national guidelines. A review just a few days later established that it was not sufficiently accurate for GPs to use in patients with suspected COVID-19, so existing online guidance needed to be rewritten to avoid patient harm (Greenhalgh, Question: Should the Roth score be used in the remote assessment of patients with possible COVID-19?, 2020).

As part of the RCGP's response to the COVID-19 pandemic, in early March the College set up a COVID-19 Resource Hub, with the intention of hosting both RCGP-generated content and external guidance. Making use of an existing Moodle 3.5 platform, the web development team was able to publish the first iteration of the Hub within a few days and subsequently respond rapidly to requests for edits or the addition of new material, usually within a matter of hours. Moodle's flexibility as a content delivery platform allowed the production of a variety of learning activities: SCORM packages, screencasts, podcasts and a variety of webinars were all used to cover the emerging knowledge. With increasing content and increased usage by health care professionals, the Moodle team was able to adapt the site daily and respond to the needs of both learner and the content developers, resulting in various design iterations of the Resource Hub over 12 weeks (see Figure 1). 

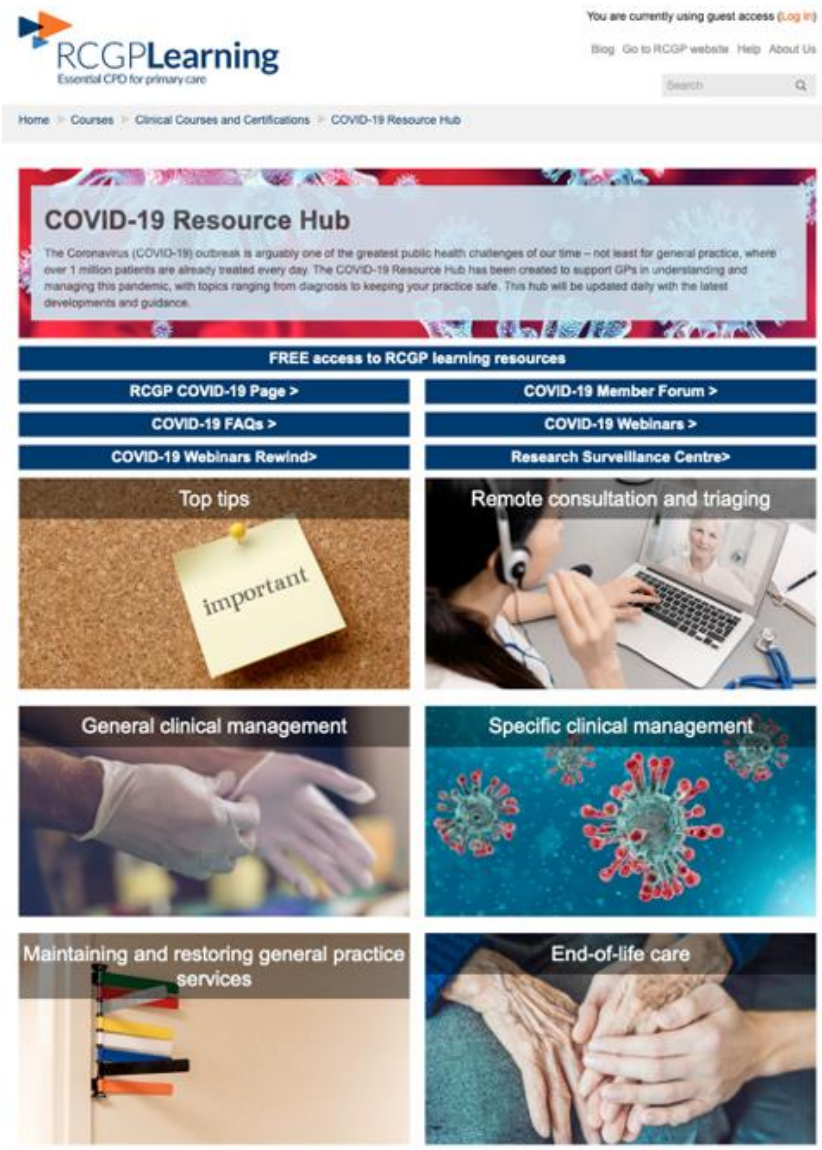

Specific patient groups and settings
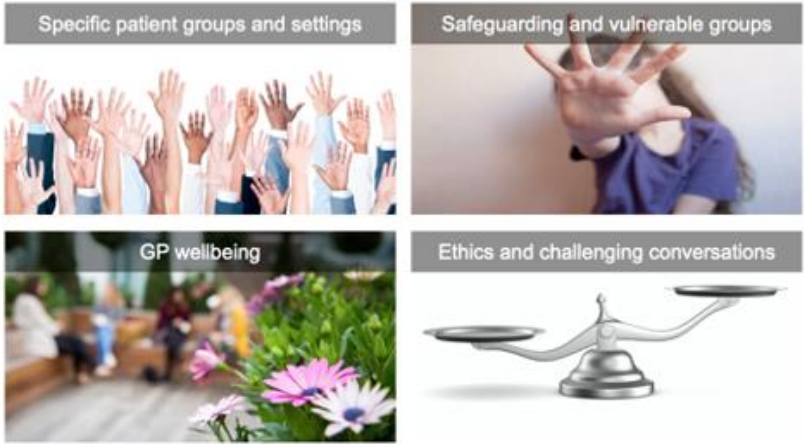

Ethics and challenging conversations
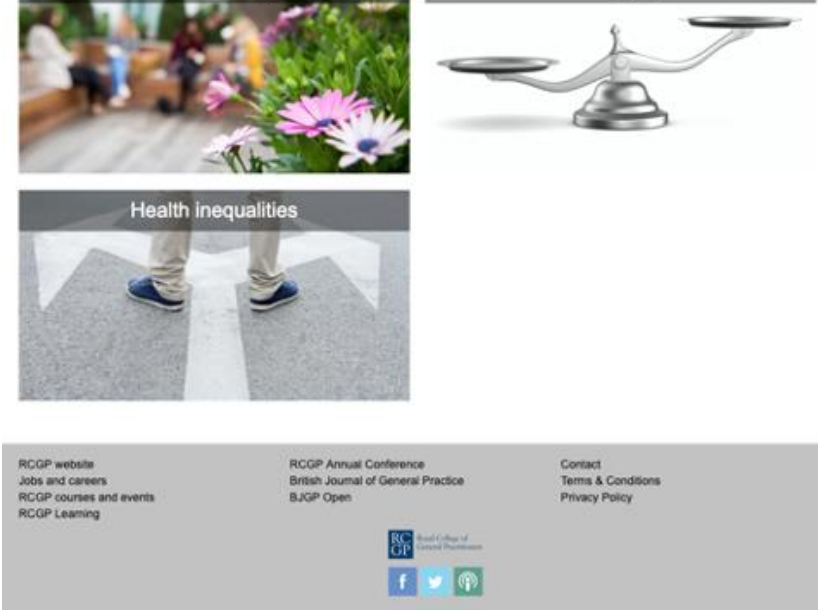

Figure 1. Screenshot of COVID-19 resource hub as of 22/06/2020 
Due to the novel nature of the COVID-19 threat, new guidance for GPs had to be produced on an unprecedented scale. This required cross-sector cooperation with other Royal Colleges and professional organisations, with initial contacts quickly generating a large volume of new guidance and content for potential publication. It quickly became apparent that the existing model of content production and quality assurance was not responsive or prolific enough to keep up with the requirements of this project, so the Resource Hub team drew in staff and clinicians from other departments across the College. In addition, clinical expertise was requested (and quickly offered) from external organisations including universities, public health agencies and research institutions. This allowed new guidance to be highlighted very rapidly and new content developed on the basis of this guidance and new evidence, as it emerged. In addition, the College's existing clinical networks allowed resources relating to specialist areas to be developed, including managing COVID-19 in secure environments, palliative care, respiratory medicine and diabetes.

The pace of delivery and the volume of work meant that the quality assurance process for the development of new materials needed to be extremely streamlined and efficient, whilst maintaining high standards of output. In addition, there was a need to ensure that any new CPD resources published under the aegis of the RCGP was aligned with the policy position of the College. A small leadership group was set up with oversight of both clinical and policy aspects of the College's COVID-19 response. This group met frequently (initially three times per week) to review proposals for new resources and check the alignment of newly-created resources prior to publication. Between these stages, the e-learning and webinar development team managed a compressed quality assurance process that produced new content within days instead of months. The previous six step quality assurance was reduced to three steps with a significant reduction in turnaround time of new content: authors were supported by experienced e-learning fellows in the development of new materials and draft resources were checked by the College's medical director for e-learning. Within two months, the newly-formed team created sixty new individual eLearning items and vetted and hosted seventy external resources, spread over 9 different subgroups. Usage of the OLE increased significantly: compared to the previous 3 months there was an increase of $170 \%$ of individual visitors to the website.

This demonstrates the importance of operational agility in response of a crisis such as the COVID-19 pandemic: department heads were able to quickly change workflows in short iterations with constant feedback from the assigned project teams. As the OLE's content management system was not outsourced or subject to a service-level agreement, the Moodle administration team was able to respond immediately to requests for changes from their clinical colleagues. Administrators worked continuously on breaking down silos, fostering collaboration across teams and made sure the relationship to external stakeholders was kept close. As a consequence of the pandemic, work in a number of areas of the College had been paused, particularly those relating to face-to-face events or clinical topics that were now a lower priority for GPs. This allowed staff to be temporarily seconded to the College's COVID-19 response, with clear areas of responsibility. Small, focused groups were established which met virtually on a frequent basis; this allowed intelligence and guidance to cascade from the College's leadership, through senior managers to the project delivery teams, and reports on progress to pass back to the leadership. The project manager for the OLE released a daily, up to date list of content that was in production which gave all stakeholders an overview over the current state of the Resource Hub and development pipeline, from the College president to project officers and administrators. Since the inception of the RCGP's COVID-19 response in early March 2020 a high level of review, reflection and iteration went into the Resource Hub project across all hierarchical levels. As the timeline of the pandemic and the College's response proceeded, processes were adapted and shifted to address both the changing circumstances and lessons learned. Teams evolved, with new individuals brought in and others released as necessary. Quality assurance and approval processes were tweaked to avoid development bottlenecks or overloading specific individuals. The focus of support shifted; for example, in early March resources relating to good practice in remote consultation were a priority, whereas by late May there was a growing need for CPD related to the respiratory management of recovering COVID-19 patients.

\section{DISCUSSION}

This case demonstrates how an environmental trigger can create rapid organisational change, both through adapting formal staffing structures and informally through negotiated changes in staff roles. These changes were not limited to organisational aspects: andragogical and educational practice had to adapt as well, with 
some paradigms needing re-evaluation. A key feature of this case, which is likely to have been experienced by other organisations, is how the critical imperatives of the pandemic forced very rapid adjustments in modes of project delivery and management. Nevertheless, there was a clear and shared vision at all levels of the organisation; an appreciation of the need to deliver results to meet the challenge; small, agile teams were drawn together whose individuals were selected for their talent, self-motivation and good will; the project was managed with rigid control systems replaced by looser, super-productive processes.

This mirrors the fundamental changes in practice that GPs were facing, which the RCGP was attempting to support. Traditionally, attempts to effect organisational change would face significant inertia and perhaps resistance; in March/April 2020 there was a universal recognition across the College for the need to work differently. Warren Buffet spoke of the "institutional imperative" as an often-unrecognised force which can resist radical learning and change to existing structures and systems (Buffet, 1989). The urgent need to provide resources and guidance for frontline GPs - within a matter of days - overrode this imperative in the case of the RCGP. The confluence of circumstances meant that the RCGP saw, almost without explicit intention, the emergence of the key principles behind what Alan Kay has described as the 'power of context' which drove the success of the Xerox PARC programme of the 1970s (Kay, 2004) and which for the RCGP created a new resource that accompanies all members of the primary care team as a reliable source of CPD through the next stages of the pandemic.

\section{ACKNOWLEDGEMENTS}

Many thanks to Damian Bardiger and Sabeen Altaf for their suggestions for this paper.

\section{REFERENCES}

Abbasi, K. (2020, May 1). The many uncertainties of COVID-19. Journal of the Royal Society of Medicine, 113(5), 167.

Ahmed, W., Vidal-Alaball, J., Downing, J., \& Lopez Segui, F. (2020, April 18). Dangerous Messages or Satire? Analysing the Conspiracy TheoryLinking 5G to COVID-19 through Social Network Analysis. Retrieved from Journal of Medical Internet Research: preprints.jmir.org/preprint/19458

Buffet, W. (1989). Letter to the shareholders of Berkshire Hathaway Inc. Retrieved from https://www.berkshirehathaway.com/letters/1989.html

Cameron, D., \& Grant, A. (2017). The role of mentoring in early career physics teachers' professional identity construction. International Journal of Mentoring and Coaching in Education, 128-142.

Cunningham, D., Alexander, A., Luty, S., \& Zlotos, L. (2019, May 8). CPD preferences and activities of general practitioners, registered pharmacy staff and general practice nurses in NHS Scotland - a questionnaire survey. Education for primary care, 30(4), 220-229.

Densen, P. (2011). Challenges and opportunities facing medical education. Transactions of the American Clinical and Climatological Association, 122, 48-58.

Fei Zhou, T. Y. (2020, March 9). Clinical course and risk factors for mortality of adult inpatients with COVID-19 in Wuhan, China: a retrospective cohort study. The Lancet.

General Medical Council (UK). (2012, June). Continuing professional development. Retrieved from General Medical Council: https://www.gmc-uk.org/-/media/documents/cpd-guidance-for-all-doctors-0316_pdf-56438625.pdf

Glaveanu, V. P. (2017). Editorial Psychology in the Post-Truth Era. Europe's Journal of Psychology, 13(3), 375-377.

Greenhalgh, T. (2020, March 20). Covid-19: a remote assessment in primary care. British Medical Journal, 368.

Greenhalgh, T. (2020). Question: Should the Roth score be used in the remote assessment of patients with possible COVID-19? Oxford: Oxford COVID-19 Evidence Service Team, Nuffield Department of Primary Care Health Sciences, University of Oxford.

Kay, A. (2004). The Power of Context: remarks upon receipt of the Draper Award, National Academy of Engineering, 24 Feb 2004. Retrieved from http://www.vpri.org/pdf/m2004001_power.pdf

MacWalter, G., McKay, J., \& Bowie, P. (2016). Utilisation of internet resources for continuing professional development: a cross-sectional survey of general practitioners in Scotland. BMC Medical Education, 16(24).

Morrow, G. e. (2014). Professionalism in healthcare professionals. HCPC. 
Moss, P., Barlow, G., Easom, N., Lillie, P., \& Samson, A. (2020, February 27). Lessons for managing high-consequence infections from first COVID-19 cases in the UK. The Lancet, e46.

Pilat, D. (2015). Access and the great socioeconomic divide. In R. Charlton, Compassion, Continuity and Caring in the NHS (pp. 15-20). London: RCGP.

Pilat, D. (2016). Content development for 72,000 Learners: an online learning environment for general practitioners; a case study. Proceedings of the international conference e-learning 2017 (pp. 28-34). Lisbon: IADIS.

RCGP. (2020, May). GP Curriculum: an overview. Retrieved from RCGP: https://www.rcgp.org.uk/trainingexams/training/gp-curriculum-overview.aspx

Royal College of General Practitioners. (2012, March). History of the College. Retrieved May 2020, from Royal College of General Practitioners: https://www.rcgp.org.uk/about-us/the-college/who-we-are/history-heritage-andarchive/history-of-the-college.aspx

Royal College of General Practitioners eLearning Department. (2017). Learning Technologies Award; Best learning technologies project public \& non profit sector; Submission. London: RCGP.

VanNieuvenborg, L., Goossens, M., De Lepeleire, J., \& Schoenmakers, B. (2016, April). Continuing medical education for general practitioners: a practice format. Postgraduate Medical Journal, 92(1086), 217-222. 